Article

\title{
Strategy and Evaluation of Vehicle Collision Avoidance Control via Hardware-in-the-Loop Platform
}

\author{
Sin-Li Chen ${ }^{1}$, Chang-Yi Cheng ${ }^{1}$, Jia-Sheng $\mathrm{Hu}^{1, *}$, Jinn-Feng Jiang ${ }^{2}$, Tsu-Kun Chang ${ }^{2}$ and \\ Hung-Yuan Wei ${ }^{2}$ \\ 1 Department of Greenergy, National University of Tainan, No. 33, Sec. 2, Shu-Lin St., Tainan 700, Taiwan; \\ air150181@gmail.com (S.-L.C.); s9003794@gmail.com (C.-Y.C.) \\ 2 Planning and Promotion Department, Metal Industries Research \& Development Center, Taipei 100, Taiwan; \\ jfjiang@mail.mirdc.org.tw (J.-F.J.); m830483@mail.mirdc.org.tw (T.-K.C.); \\ davidwei@mail.mirdc.org.tw (H.-Y.W.) \\ * Correspondence: jogson@ieee.org; Tel.: +886-6-260-5051
}

Academic Editors: Stephen D. Prior and Teen-Hang Meen

Received: 20 September 2016; Accepted: 27 October 2016; Published: 1 November 2016

\begin{abstract}
This paper proposes a novel control approach for vehicle collision avoidance of urban vehicles. For safe driving in urban environments, this paper presents both one-dimensional and two-dimensional solutions, which can be applied to the collision avoidance via steering assistance, automatic braking, and warning of collision. Strategies are verified under the software CarSim, and the experimental evaluations are carried out under the combination of CarSim with a hardware-in-the-loop platform. The results show the feasibility and effectiveness of the proposed algorithm on vehicle collision avoidance.
\end{abstract}

Keywords: collision avoidance system; adaptive cruise control; fuzzy control; autonomous emergency braking; hardware-in-the-loop

\section{Introduction}

Recently, steering assistance technologies of the vehicle have been on the rise because of safety and legal requirements. Automakers are now urgently developing and announcing relevant safety functions, such as autonomous emergency braking (AEB), to highlight steering assistance. Basically, AEB is a kind of technology for collision avoidance systems (CASs). AEB can be treated as a one-dimensional CAS. However, a general CAS should cover not only one-dimensional collision avoidance, but also two-dimensional collision avoidance. Consequently, the CAS is a necessary function for steering assistance. According to Fildesa et al. [1], the rear-end collision is $32 \%$ of all the car accidents in urban cities, especially in crowded traffic. Additionally, their results showed that by merely adding the AEB function, rear-end collisions can be effectively reduced by $38 \%$. It is known that AEB is a one-dimensional approach for the CAS. However, in some city driving scenarios, certain highway driving is still required. Therefore, the two-dimensional CAS sparks the interest of this study. Generally, the presented CAS includes both one-dimensional and two-dimensional collision avoidance technology. The one-dimensional approach is similar to the AEB and the known adaptive cruise control (ACC). In addition, the two-dimensional approach is the basis of automatic steering. The CAS approaches are known as the advanced driver assistance systems (ADAS). They can decrease the fatigue of the driver and the possibility of accidents [2]. For instance, when the ADAS prompt is considered as the distance up to the maximum, the driver does not pay any attention to it. If the distance is less than the minimum, the system will initiate one-dimensional braking automatically to avoid rear-end collisions. However, if there are unexpected obstacles or accidents that occur while 
driving, the system intervenes to avoid the collision. Therefore, the intelligence for driving intervention based on ADAS is important [3].

Basically, the embedded system needs to intervene with control measures in a timely manner and thus utilizes two judgment principles: (a) avoid all collisions and (b) never perform a faulty intervention $[4,5]$. If the system causes a disturbance between the driver and the driving safety system of ADAS, it can cause an accident. Hence, the driving strategy prompts the driver to pay attention to the distance between vehicles when it is up to the maximum. Likewise, if it is less than the minimum, the system will intervene to avoid the collision by braking. The concept is similar to that employed in some intelligent control theories. This paper proposes a fuzzy-based control algorithm to maintain the braking force and its related safety. For evaluation, testing related strategies under real conditions leads to significant efforts and costs [6,7]. In this study, a hardware-in-the-loop (HIL) platform is used for evaluations. The inputs from the operator are real; however, the vehicle dynamics are virtual. This platform setup facilitates cost-effectiveness for full-scale evaluations. In this paper, these tools are employed to evaluate the control strategy and its corresponding performances.

\section{Role of the CAS}

Because of competition, almost all new commercial vehicles have AEB as an available optional function. AEB can automatically intervene in driving when a collision is expected to happen. According to Hu et al. [8], about $40 \%$ of traffic accidents are caused by locked braking. When the brake of a vehicle is locked, the vehicle will slide and cause a rear-end collision due to the traction loss and inertia force. Hence, the anti-lock braking system (ABS) was proposed to improve the problem. It is known that the traction control system (TCS) is also an anti-skidding function. Consequently, the ABS and TCS are good foundations of one-dimensional AEB and the CAS. The two-dimensional CAS involves the turning scenario. Consequently, the electronic stability control (ESC) is a required function. The ESC is an active safety technology that improves a vehicle's dynamic stability by detecting steering deterioration and supporting the regaining of vehicle control $[9,10]$. Hence, the ESC does not protect occupants in a crash but helps to prevent abnormal steering in the first place. Definitely, for the two-dimensional CAS, the ESC is a crucial function. Without the basis of ESC, the two-dimensional CAS may still unavailable. The following Figure 1 shows the relationship between the mentioned technologies.

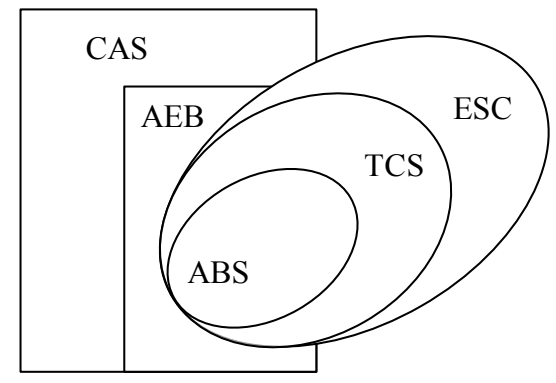

Figure 1. Relationship between all technologies of vehicle stability assistance.

\section{Proposed System}

The timing of slamming on the brakes is according to the relative velocity and distance of the two vehicles. The rear-end collision system is usually evaluated according to the maximum distance. If the distance achieves the warning zone, the system will only initiate an alarm to the driver. Conversely, if it achieves the minimum limit, the system actively controls the vehicle to brake to avoid the collision. Figure 2 shows the detection of the front and rear cars. 


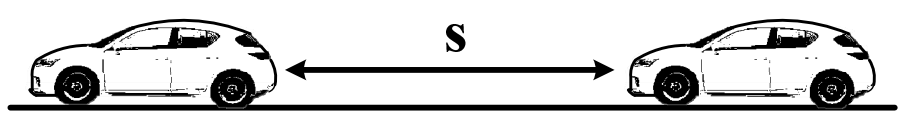

Figure 2. Distance from the front to rear.

The key to one-dimensional AEB is the relative velocity $v_{r}$, the deceleration $a^{\prime}$ of the front car, and the maximum braking acceleration $a_{r}$ of the rear car. Note that $v_{r 0}$ is the triggering speed, $v_{f}$ is the ground speed of the front car, $s$ is the relative distance of the two vehicles. Therefore, the related motion equations are as follows:

$$
\begin{gathered}
v_{r}^{2}=v_{r 0}^{2}+2 a_{r} s \\
a^{\prime}=\frac{d v_{f}}{d t}
\end{gathered}
$$

Note that $\left|a_{r}\right|>\left|a^{\prime}\right|$ and the target is $v_{r} \approx 0$. In addition, the braking deceleration cannot take place beyond the maximum deceleration of the AEB. Otherwise, the traction force saturation will occur because of the nonlinear behavior of the tire's dynamics. Safety is the first issue. The distance $s^{\prime}$, which is the collision avoidance zone, is set as

$$
s^{\prime}=-\frac{v_{r 0}{ }^{2}}{2 a_{r}}
$$

Then, according to the distance $s^{\prime}$, the system can invasively initiate emergency braking. However, the one-dimensional automatic brake causes different saturated and slippery tire conditions against the different velocities and distances of the front vehicle; therefore, it is immediately difficult to measure and compensate for. Table 1 gives the proposed fuzzy limits. Under this table, the rear vehicle can have a performance that is more comfortable while the AEB is activated. This rule is based on a series of engineering tests and adjustments. Safety and comfort are the dilemmas of the one-dimensional automatic brake. Therefore, steering to avoid collision from two-dimensional motion control is then proposed. It improves the driving safety and energy efficiency. Figure 3 shows the vehicle changing lanes to avoid a collision. When steering to avoid a two-dimensional collision begins, the vehicle requires one or two instances of steering. Therefore, it will cause significant yaw moment. The vertical and horizontal tractive forces of the tires rapidly decline due to nonlinear saturation in case the vehicle is out of control and in danger of losing its tractive force when steering. Therefore, control is the key to avoiding two-dimensional collisions. Figure 4 gives the model of the vehicle engaged in two-dimensional motion, and the vehicle of the motion equation is shown the following formula:

$$
\begin{gathered}
M a_{y}=M V_{x}(\gamma+\dot{\beta})=2 C_{f}\left(\delta_{f}+\frac{l_{f}}{V_{x}} \gamma-\beta\right)-2 C_{r}\left(\frac{l_{r}}{V_{x}} \gamma+\beta\right) \\
N_{i n}=I_{n} \dot{\gamma}=2 l_{f} C_{f}\left(\delta_{f}+\frac{l_{f}}{V_{x}} \gamma-\beta\right)-2 l_{r} C_{r}\left(\frac{l_{r}}{V_{x}} \gamma+\beta\right)+N_{z}+N_{d}
\end{gathered}
$$

where $\beta$ is the side slip angle, $\gamma$ is the yaw-rate of the center of the vehicle's mass, $M$ is its mass, $a_{y}$ is the lateral acceleration, $\delta_{f}$ is the steering angle of the front wheels, $V_{x}$ is the longitudinal velocity of the vehicle, $I_{n}$ is the nominal moment of inertia. $C_{f}$ and $C_{r}$ are the cornering stiffness of the front and rear wheels. $l_{f}$ and $l_{r}$ are the wheelbase of the center of the front and rear wheels. $N_{z}$ is the active compensation torque. $N_{d}$ is the torque from environmental disturbances, such as crosswind. Let

$$
\begin{gathered}
N_{t}=2 l_{f} C_{f}\left(\delta_{f}+\frac{l_{f}}{V_{x}} \gamma-\beta\right)-2 l_{r} C_{r}\left(\frac{l_{r}}{V_{x}} \gamma+\beta\right) \\
N_{i n}=\left(N_{t}+N_{d}\right)+N_{z}=N_{t d}+N_{z} \\
N_{t d}=N_{t}+N_{d}
\end{gathered}
$$


Table 1. Braking conditions for one-dimensional CAS (collision avoidance system).

\begin{tabular}{cccc}
\hline Strategy Map & $\boldsymbol{s}^{\prime}<\mathbf{2 0 ~ \mathbf { m }}$ & $\mathbf{2 0} \leq \boldsymbol{s}^{\prime}<\mathbf{4 0} \mathbf{m}$ & $\mathbf{4 0} \leq \boldsymbol{s}^{\prime}<\mathbf{6 0} \mathbf{m}$ \\
\hline$v_{r}<30 \mathrm{~km} / \mathrm{h}$ & $60 \%$ Braking & $50 \%$ Braking & $40 \%$ Braking \\
$30 \leq v_{r}<60 \mathrm{~km} / \mathrm{h}$ & $80 \%$ Braking & $70 \%$ Braking & $60 \%$ Braking \\
$v_{r} \geq 60 \mathrm{~km} / \mathrm{h}$ & $100 \%$ Braking & $90 \%$ Braking & $80 \%$ Braking \\
\hline
\end{tabular}

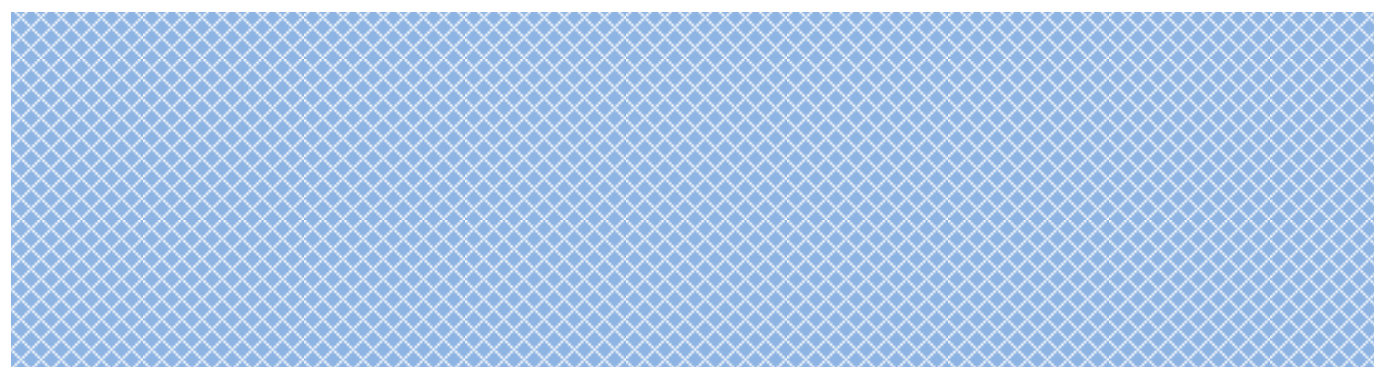

Figure 3. Schematic of the vehicle changing lanes to avoid collision.

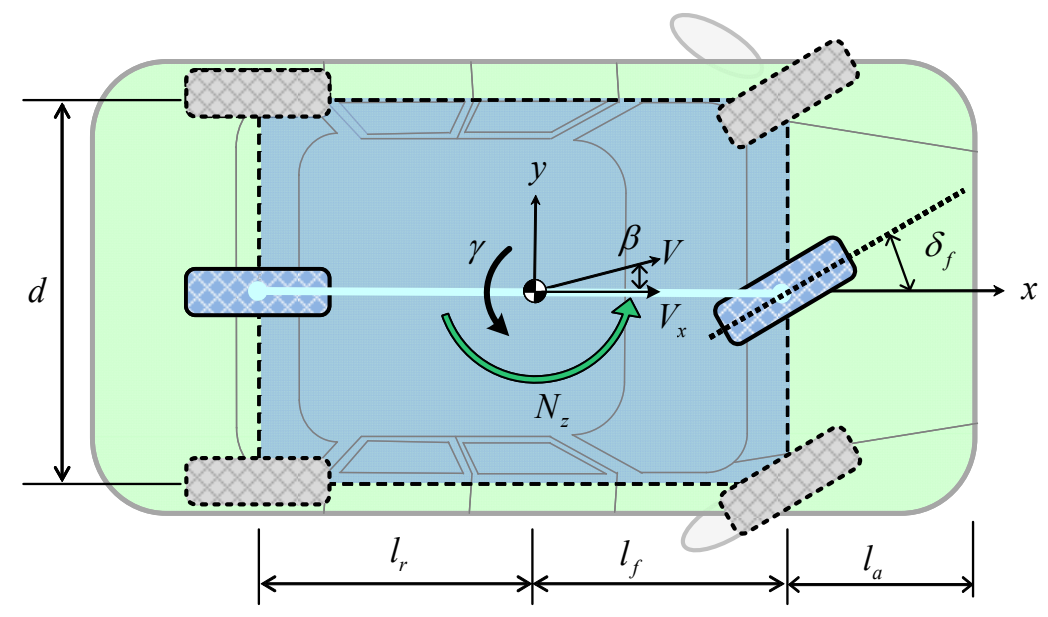

Figure 4. Motion model of vehicle.

This paper proposed a solution to deal with two-dimensional CAS. Note that when the rear vehicle overtakes the front one by steering to avoid two-dimensional collisions, $r_{f}$ and $r_{r}$ are the safe radius of the front and rear cars, and $r_{\min }$ is the minimum radius of the rear steering. When the distance between vehicles is $0.5 r_{\min }$, the system will automatically steer the steering wheel to avoid the collision by $30^{\circ}$ or $45^{\circ}$, provided that the adjacent lane is clear. The steering schematic is shown in Figure 5. Generally, the minimum steering radius follows the formula of geometric relationships.

$$
r_{\min }=\sqrt{\left(L+l_{a}\right)^{2}+L \cot ^{2} \delta_{f}}
$$

Herein lies the equation $L=l_{f}+l_{r} ; l_{a}$ is the distance from the front bumper to the front wheels. The yaw moment can be controlled by the ESC. Stable steering of the vehicle produces the order of the yaw rate as

$$
\gamma^{*}=\frac{k V_{x}}{\tau s+1} \delta_{f}
$$

Here, $k$ is the adjustable gain and $\frac{1}{1+\tau s}$ is the low-pass filter. In this scheme, the yaw moment control should be guaranteed. Consequently, the ESC should be activated all the time. Otherwise, the vehicle may fall into an unstable scenario when performing an overtaking. 


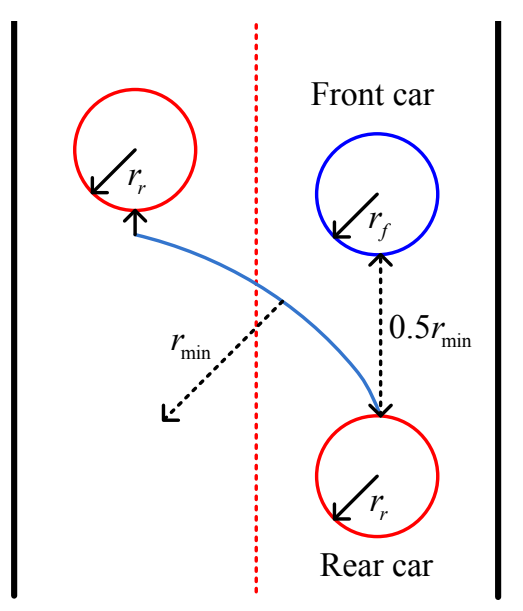

Figure 5. Steering basis of vehicle.

In this paper, the two-dimensional CAS is called the dynamic collision avoidance control (DCAC). It combines all the mentioned technologies in a dynamic situation. Figure 6 shows the flowchart of the presented system. As can be seen in this figure, the presented system covers the functions of ACC and AEB. Additionally, the DCAC can achieve collision avoidance in a dynamic way. Thus, it appears to be an overtaking scenario; the rear vehicle should change its lane once or twice. Evaluations in the following section will be carried out to verify the proposed algorithm. Note that it has the potential to be employed on the Intelligent Transportation Systems (ITSs). For a wheeled vehicle with tires, our proposed approach, namely the DCAC, can be applied to trucks, buses, and even linked vehicles, such as railway trains. The proposed approach can be a foundation for modern autonomous vehicles. It endows the autonomous vehicles with nimble and reliable reactions on motion and safety regarding collision avoidance. Consequently, for further investigations at the next stage, the proposed approach can be applied to a full-scale autonomous vehicle for ground tests.

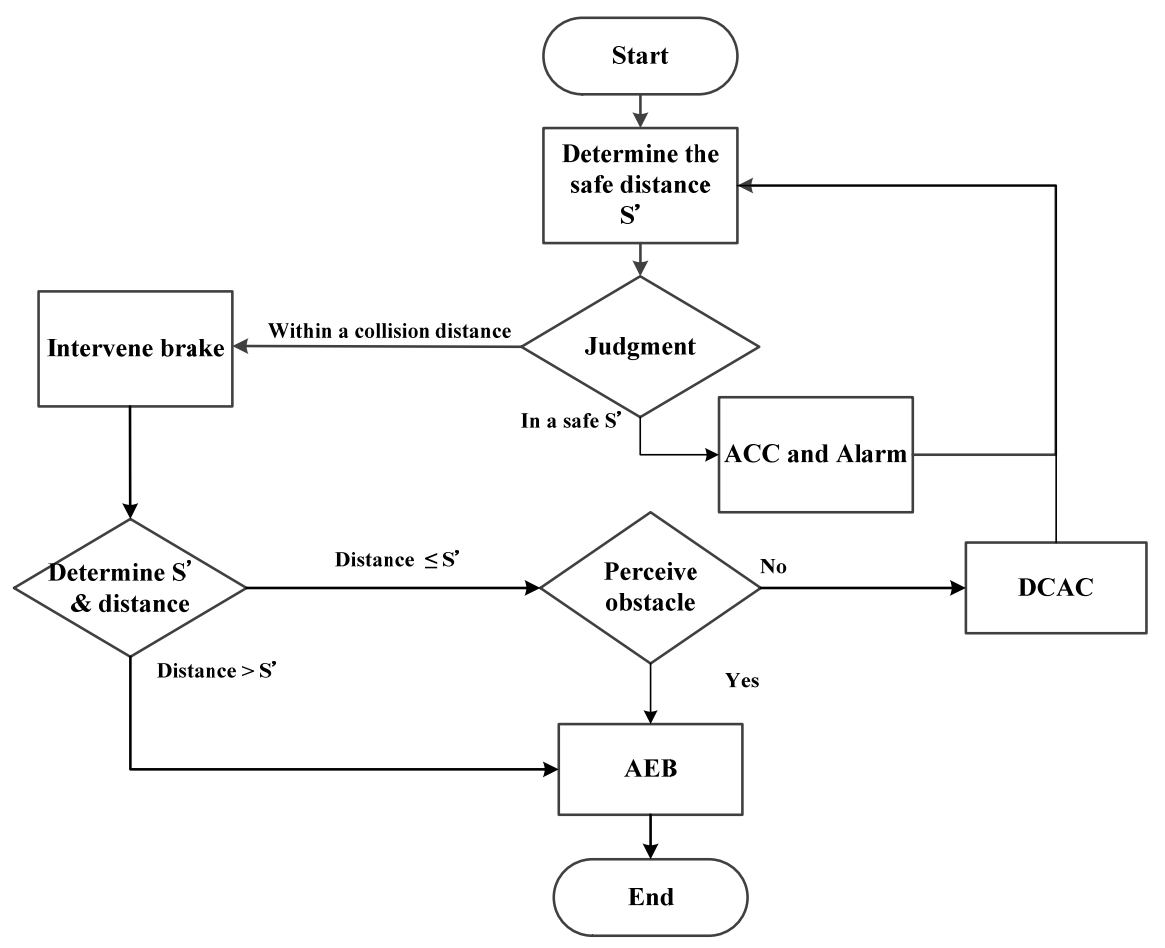

Figure 6. Flowchart of the proposed approach. 


\section{Evaluations with Hardware-in-the-Loop}

The proposed approach was verified under the software CarSim (Version 8.03, Mechanical Simulation Corporation, Ann Arbor, MI, USA, 2012) with hardware-in-the-loop (HIL) packages. CarSim can provide the virtual model of the vehicles and simulate the conditions of different roads. The HIL provides the steering command from the operator and software to make some experimental scenarios. The commands such as steering the wheel and pedal are input directly from the hardware. In this paper, CarSim with the HIL setup is utilized to verify the correctness of the presented issues. The results combine both virtual vehicle model dynamics and real hardware inputs. The evaluations facilitate the cost-effectiveness on the experimental evaluations. Note that the HIL evaluation was mainly built for the purpose of testing different scenarios on real steering conditions. Hence, part of the simulation can employ real and/or virtual devices in order to achieve a cost-effective and safe experimental environment. No doubt the HIL can evaluate an engineering problem in a cost effective way, especially for severe and aggressive steering tests.

Figure 7 shows the evaluation results of the one-dimensional AEB at $50 \mathrm{~km} / \mathrm{h}, 70 \mathrm{~km} / \mathrm{h}$, and $90 \mathrm{~km} / \mathrm{h}$, respectively. As can be seen in this figure, the proposed fuzzy braking has a better performance on stopping. In addition, the steering required to avoid two-dimensional collisions is built on the one-dimensional automatic brake. Basically, the AEB is activated when the distance is too short. When the distance reaches the preset value, the system will intervene to brake and avoid the collision. If the system suffers a disturbance from sudden accidents or intrusions to the brake intervention distance, it has to steer to avoid the obstacle. However, this function needs to consider the surroundings to determine the feasibility of intervention steering. If the surroundings allow for such maneuvers, the vehicle can automatically steer to avoid collisions.

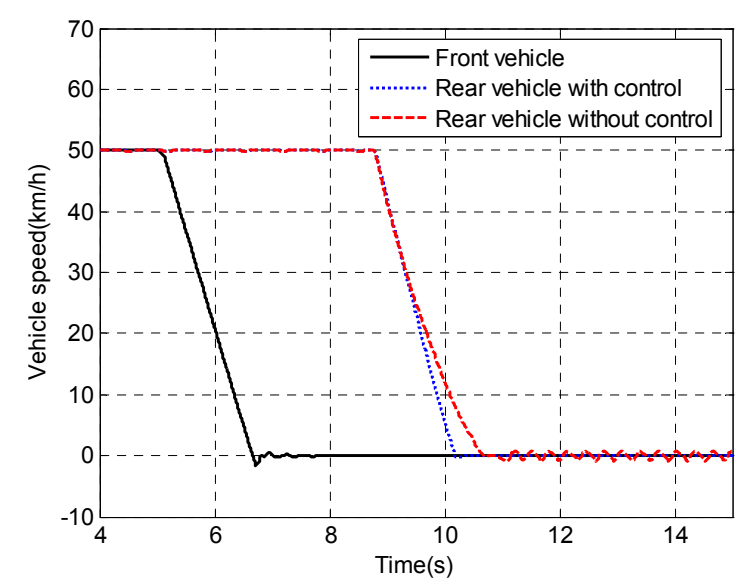

(a) At $50 \mathrm{~km} / \mathrm{h}$.

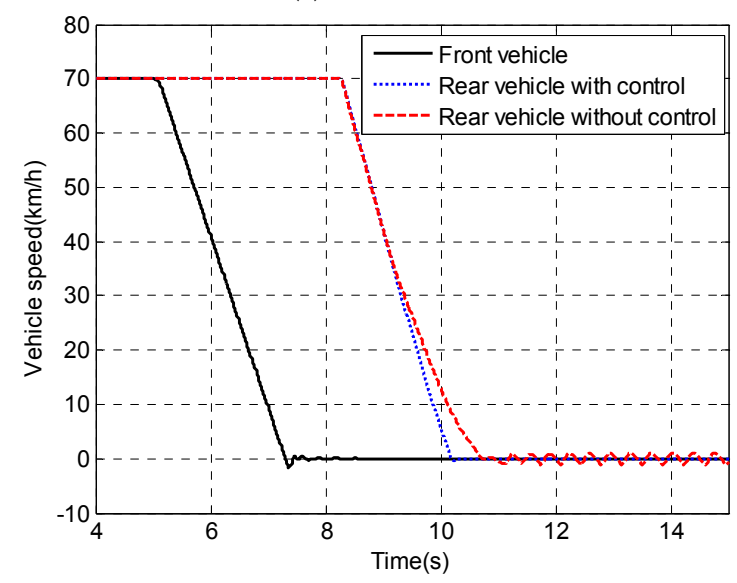

(b) At $70 \mathrm{~km} / \mathrm{h}$.

Figure 7. Cont. 


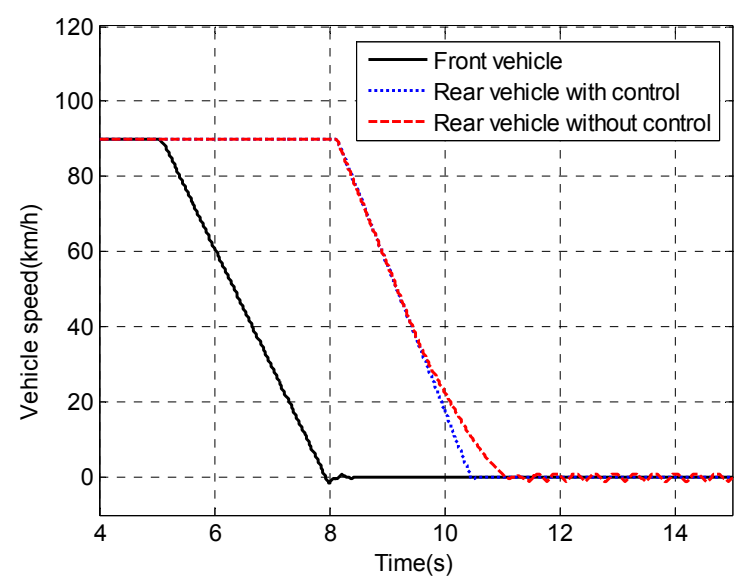

(c) At $90 \mathrm{~km} / \mathrm{h}$

Figure 7. Velocity profile of braking.

Likewise, the evaluation of the two-dimensional collision uses three velocities as the simulated conditions: $50 \mathrm{~km} / \mathrm{h}, 70 \mathrm{~km} / \mathrm{h}$, and $90 \mathrm{~km} / \mathrm{h}$. When the distance is too short to initiate one-dimensional braking automatically, steering to avoid two-dimensional collisions will be executed, as shown in Figure $8 \mathrm{a}$, where two vehicles driving in the same lane simulate the steering to avoid two-dimensional collisions. This condition is simulated by two vehicles tracking each other. If the front vehicle suffers an accident or an emergency, the brake command will be given to both vehicles. The rear vehicle automatically initiates one-dimensional braking to prevent the collision; however, the condition is an unexpected accident, and thus it assesses the relative distance between the vehicles as being too short to avoid a collision by solely employing one-dimensional braking. Therefore, the system will start steering to avoid a collision by DCAC. Figure $8 \mathrm{~b}$ shows the photo taken from the experimental evaluation based on HIL where the user inputs commands via a real steering wheel and pedal. The virtual side from CarSim represents an animation for simulating the real driving experience. Figure 9 shows the speed profile of the proposed steering. Figure 10a shows the wheel steering angle under the steering wheel rapidly turning $30^{\circ}$ or $45^{\circ}$. Note that the reduction ratio of the steering angle between the operator and front wheels is $20: 1$. Figure $10 \mathrm{~b}$ demonstrates the dynamic stability by revealing the side slip angle during the lane change. As can be seen in this figure, the system stability is dominated by ESC when the DCAC is activated.

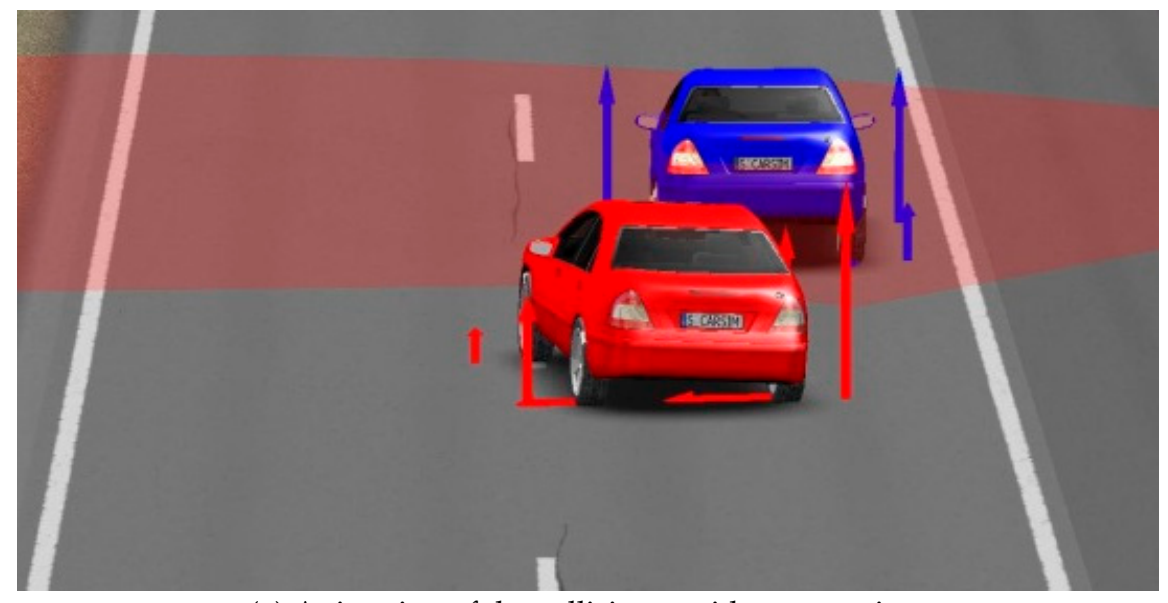

(a) Animation of the collision avoidance steering.

Figure 8. Cont. 


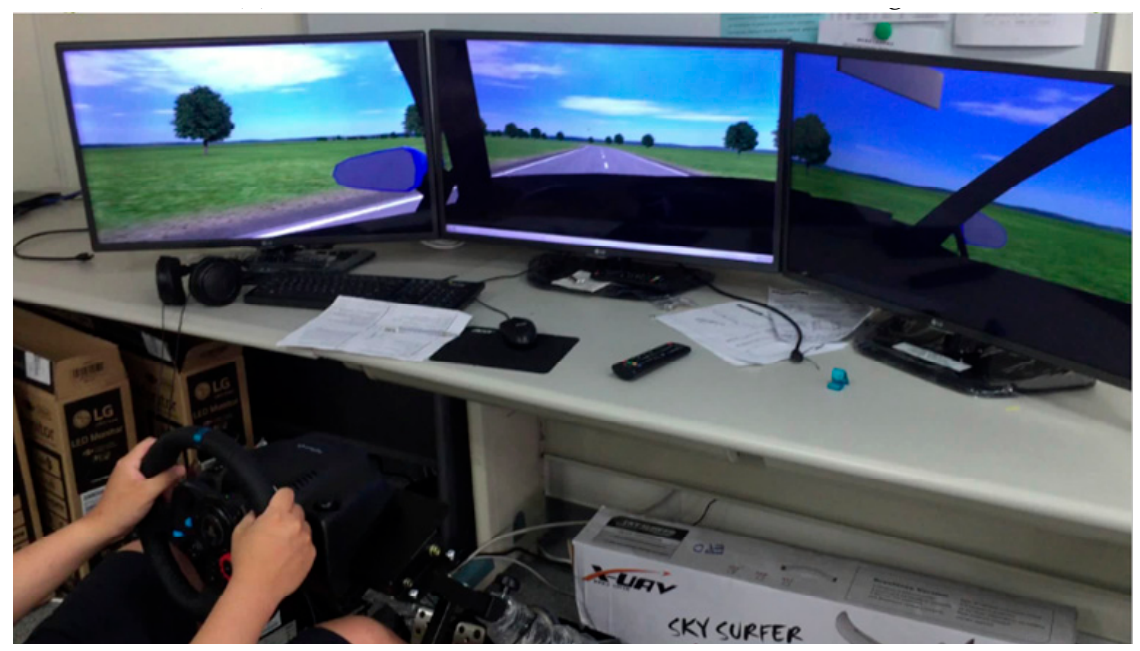

(b) Evaluation via HIL (hardware-in-the-loop).

Figure 8. Environmental setup of the evaluations.



(a) At $50 \mathrm{~km} / \mathrm{h}$.

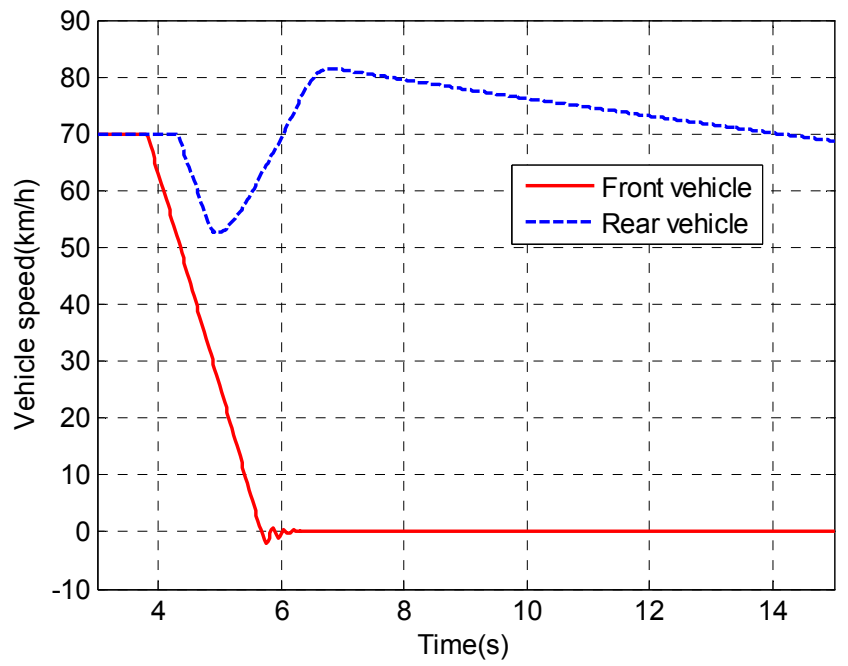

(b) At $70 \mathrm{~km} / \mathrm{h}$.

Figure 9. Cont. 


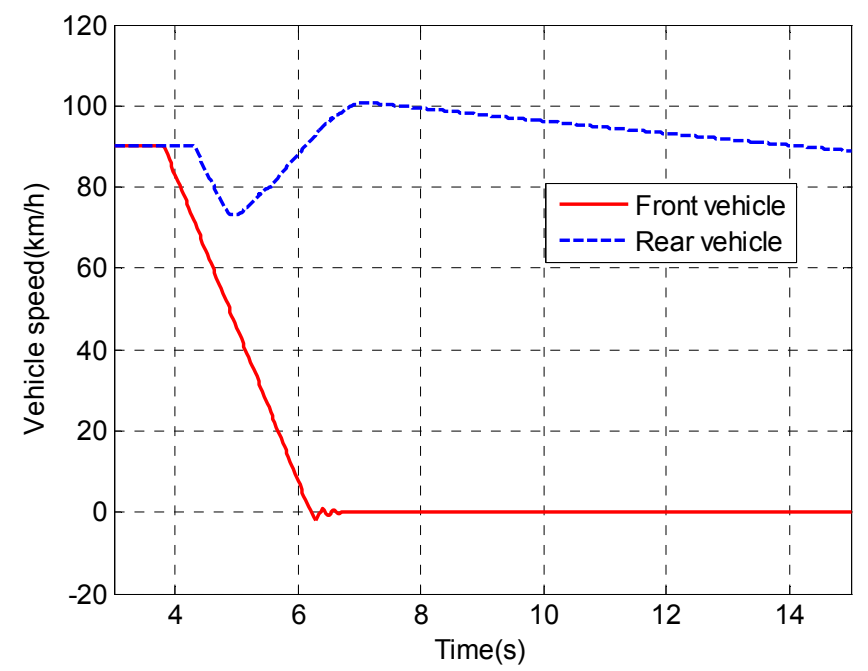

(c) At $90 \mathrm{~km} / \mathrm{h}$.

Figure 9. Velocity profile of the steering.



(a) Wheel steering angle.

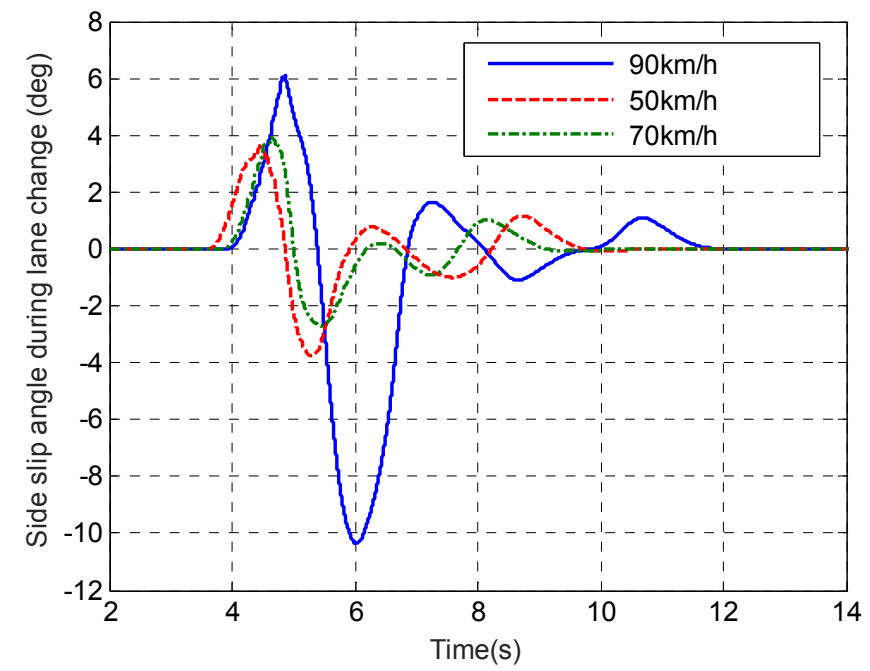

(b) Side slip angle during lane change.

Figure 10. Wheel steering angle and side slip angle. 


\section{Conclusions}

Preventing one-dimensional vehicle collisions is key to ACC and AEB. Two-dimensional automatic collision avoidance is based on the one-dimensional model and is considered as one of the automatic driving technologies. This study is based on preventing one-dimensional rear-end collisions and deriving the automatic collision avoidance of the two-dimensional approach. The control algorithm and intervention steering have decreased the possibility of vehicle collisions by effectively helping vehicles avoid unexpected obstacles. This paper has implemented both one- and two-dimensional collision avoidance strategies under the evaluation of HIL. The results have verified that the feasibility and effectiveness of the proposed intelligent algorithm are successful for vehicle collision avoidance.

Acknowledgments: This work was supported by Metals Industry Research and Development Center (MIRDC) of Taiwan and Ministry of Science and Technology (MOST) of Taiwan, under projects MIRDC-0803-V101(104), MOST 104-2622-E-024-003-CC 2, and MOST 104-2218-E-006-026.

Author Contributions: S.-L.C., C.-Y.C. and J.-S.H. initiated and discussed the research problem; J.-S.H., J.-F.J., T.-K.C., and H.-Y.W. conceived and developed the methods; S.-L.C. and C.-Y.C. performed the simulations, experiments, and took photos; S.-L.C. and J.-S.H. analyzed the data; C.-Y.C. and J.-S.H. prepared and wrote the paper.

Conflicts of Interest: The authors declare no conflict of interest.

\section{References}

1. Fildesa, B.; Keallb, M.; Bosc, N.; Lied, A.; Pagee, Y.; Pastorf, C.; Pennisig, L.; Rizzih, M.; Thomasi, P.; Tingvallj, C. Effectiveness of low speed autonomous emergency braking in real-world rear-end crashes. Accid. Anal. Prev. 2015, 81, 24-29. [CrossRef] [PubMed]

2. Guo, C.; Meguro, J.; Kojima, Y.; Naito, T. A multimodal ADAS system for unmarked urban scenarios based on road context understanding. IEEE Trans. Intell. Transp. Syst. 2015, 16, 1690-1704. [CrossRef]

3. Butakov, V.; Ioannou, P. Personalized driver/vehicle lane change models for ADAS. IEEE Trans. Veh. Technol. 2015, 64, 4422-4431. [CrossRef]

4. Jansson, J.; Ekmark, J.; Gustafsson, F. Decision making for collision avoidance systems. SAE Tech. Pap. 2002. [CrossRef]

5. Kim, J.; Park, C.; Hwang, S.; Hori, Y.; Kim, H. Control algorithm for an independent motor-drive vehicle. IEEE Trans. Veh. Technol. 2010, 59, 3213-3222. [CrossRef]

6. Hassan, B.; Berssenbrügge, J.; Qaisi, I.A.; Stöcklein, J. Reconfigurable driving simulator for testing and training of advanced driver assistance systems. In Proceedings of the 2013 IEEE International Symposium on Assembly and Manufacturing, Xi'an, China, 30 July-2 August 2013.

7. Liu, J.; Zhang, L.; Xiao, S.; Xin, X. Development of virtual drive HILS system based on VR and CarSim. In Proceedings of the 33rd Chinese Control Conference, Nanjing, China, 28-30 July 2014.

8. Hu, J.-S.; Huang, Y.-R.; Hu, F.-R. Development of traction control for front-wheel drive in-wheel motor electric vehicles. Int. J. Electr. Hybrid Veh. 2012, 4, 344-358. [CrossRef]

9. Yin, D.; Hu, J.-S. Active approach to electronic stability control for front-wheel drive in-wheel motor electric vehicles. Int. J. Automot. Technol. 2014, 15, 979-987. [CrossRef]

10. Hu, J.-S.; Lin, X.-C.; Yin, D.; Hu, F.-R. Dynamic motion stabilization for front-wheel drive in-wheel motor electric vehicles. Adv. Mech. Eng. 2015, 7, 1-11. [CrossRef]

(C) 2016 by the authors; licensee MDPI, Basel, Switzerland. This article is an open access article distributed under the terms and conditions of the Creative Commons Attribution (CC-BY) license (http://creativecommons.org/licenses/by/4.0/). 